\title{
A Classical Physics Approach for Space Expansion: The Hubble Drift
}

\author{
Ogaba Philip Obande ${ }^{1}$ \\ ${ }^{1}$ Department of Chemistry, Ahmadu Bello University, Zaria, Nigeria \\ Correspondence: Ogaba Philip Obande, Retired, Department of Chemistry, Ahmadu Bello University, Zaria, \\ Nigeria.
}

Received: February 19, 2021

Accepted: March 31, 2021

Online Published: November 1, 2021

doi:10.5539/apr.v13n2p39

URL: https://doi.org/10.5539/apr.v13n2p39

\begin{abstract}
Accelerating expansion of metric space AEMS is investigated with classical Newtonian mechanics. Relying on earlier positions, the results are analyzed to reveal what could be a new understanding of the theoretical framework of the subject. Notably, it is shown that space is physical; it comprises aggregated waveforms of the chemical elements and shares identical quantization, periodicity and mass-evolution with matter. Three plausible methods are identified for classical investigation of the Hubble effect, all three give same result, $H_{o}=$ $49.5 \mathrm{~km} \mathrm{~s}^{-1} \mathrm{Mpc}$. AEMS results from coupling of light's $36.9^{\circ}$ tangential component $\left(v_{r}=0.75 \mathrm{r} \omega\right)$ to periodic space, i.e., a component of the vacuum field's e-m radiation couples to logarithmically decreasing distance scales, $v_{r(E)} / d_{r(E)}$, to create an acceleration relative to space not time. Multiplicity of the Hubble constant $a H_{o}$ is traceable to corresponding multiplicity of universes nested within our universe. Mass ejection from a cosmic quantum envelope is the cosmic equivalence of radioactivity, it signals ageing and eventual disappearance of the host periodic envelope from visibility. Reality is an imperturbable (ideal) Steady-State, observations thought to invalidate this view are hugely misinterpreted, an explosion in or of spacetime marking the beginning of time could not conceivably sustain, over the aeons, an accelerating expansion of metric space; furthermore, the cosmic microwave background is the zero-point energy or vacuum radiation. The active galactic nucleus or black hole is not a singularity, it is a two-way valve that facilitates circulation of mass-energy matrices across the four phases or ref. frames of reality. There is no new creation of space or matter, only continuous recycling in line with NASA's recent observation.
\end{abstract}

Keywords: atomic e-m harmonic oscillation, black hole, cosmic space expansion, physical space, wave-particle duality

\section{Introduction}

In 2017 we reported on the 'Static Sky', "a map of the entire sky, as seen from the Pan-STARRS I telescope in Hawaii," Obande (2017a). We interpreted the map an observational evidence of reality's wave-particle dual nature; in this context, reality is essentially a macrocosmic atom comprising integral wave and particulate forms, it shares common quantum characteristics with the atom. Based on this understanding, we posited that “... the 'Static Sky' has the potential to resolve all, pending and yet to be contemplated, questions in physics ... So, how does evidence for wave-particle dual nature of reality answer all existing and conceivable questions in physics? Simple, it calls for a major review of the conventional fundamental approach. ...now we have observational evidence that presents the complete picture; it merges cosmology, astrophysics and atomic physics into a single profound physical discipline that identifies with same classical Newtonian physics which differentiates only in values of extensive properties". The "Hubble Constant Crisis" provides an excellent platform upon which to put to test our understanding of the cosmic image presented in the 'Static Sky'.

We assume that, on all scales from the atom to the cosmos, reality comprises integral wave and particulate forms in which the particle is the wave-condensate encased within the wave. Furthermore, we assume also that a specific e-m harmonic oscillation defines the chemical element; in other words, the atom is a quantum e-m oscillator. It follows from these two fundamental assumptions that one could evaluate the atom's oscillation parameter or physical property if given its specific e-m oscillation frequency $v$, Obande (2017a). We rely on the 
literature, Russell \& Russell R\&R (1981), to generate $v$ values of the elements, Obande (2015a); equipped with $v$ and rest mass $\mathrm{m}$ values, we proceeded to evaluate SHM parameters of the atom's wave and particulate forms, Obande $(2015 b ; 2015 c)$. Further investigation of these waveforms reveals a physical vacuum consisting of only aggregated atomic waveforms; guided by de Broglie's pioneering studies we are now able to make a quantitative distinction between the atom's uncondensed $v_{w}$ and condensed (de Broglie) $v_{p}$ matter waves. Uncondensed matter wave manifests the familiar vacuum radiation associated with light, $r \omega=c_{o}$, it is characterized by ultra-low atomic mass $m_{w}$ values with total cosmic density $\rho_{w}^{*}=2.61 \times 10^{-36} \mathrm{~kg} \mathrm{~m}^{-3}$, Obande (2019a); on the other hand, condensed matter field, $c^{o}=3.715352291 \times 10^{-14} \mathrm{~m} \mathrm{~s}^{-1}$, manifests "relative" atomic mass $m_{p}$, Obande (2015a). Mutual correlation of SHM parameters within a particular atomic form turned up quite a few surprises: the correlation coefficients identify with observational fundamental physical constants while the exponents give quantitative measures of geometry of the coupled space, Obande (2016a; 2017b 2017c). Notably, the revelation that fundamental physical constants result from correlation of atomic waveforms' physical parameters would suggest that the visible world, comprising clusters, galaxies, stars, satellites, et cetera, is encased within electron waveform pockets, $r_{e(w)}=1.499792458 \times 10^{8} \mathrm{~m}$. If, therefore, the Hubble $H_{o}$ was truly a fundamental physical constant its (theoretical) value would emerge as coefficient of correlation of relevant parameters of the cosmic vacuum field CVF; here, we investigate the prospects within the context of classical spacetime.

\subsection{Causality of Metric Space Expansion}

Every object self-expands; it arises from harmonic-oscillation-motivated rotation of the quantum energy packet or body, Obande (2019b). Condensed matter rotation is implicated in the expansion of galaxies, Wall (2018); stars, Apell (2008); satellites, Diaz (2018); atomic mass evolution, Obande (2016b); and radioactivity, Obande (2015b). Vacuum energy density $\rho_{w}$ couples with the stress field $\sigma_{w}$ to produce an invariant vacuum radial velocity field $v_{r}$, as we shall see, the coupling of this field to intrinsic spatial periodicity causes observational accelerating expansion of metric space. Notably, the acceleration is a velocity-space, i.e., $d v / d x$, not velocity-time gradient. One discovers in the course of evaluating the chemical element's intrinsic $v$ that the generalized wave-particle energy equation or classical mass formula CMF should read, Obande (2016b; 2013),

$$
\phi h v=m c^{2}
$$

where the numerical coefficient $\phi=1.0 /$ atom for the waveform and 1.0172/atom for particulate matter. It is not usually realized that (1) accords equal wave $(h v)$ and particulate $\left(m c^{2}\right)$ nature to the atom; in other words, the atom (hence, the cosmos) is intrinsically a wave/particle composite much like the lint and seed of unginned cotton. Since the particle (i.e., seed) constitutes the material world the wave (i.e., lint) necessarily constitutes the cosmic vacuum field CVF. Empirical proof of this position, Obande (2019a; 2016a; 2017b; 20127c) implies that space shares identical quantization, periodicity and mass evolution characteristics with matter. Beginning with an account of the CVF, the rest of the paper investigates the preceding underlining positions.

\subsection{The Cosmic Vacuum Field: Composition, Structure, Properties}

Observational evidence does not support conventional notion of the cosmic vacuum field. The field is far from what consensus opinion presumes, it is as physical as a chunk of steel; its composition, structure and properties are considered in the context of the CMF.

\subsubsection{Composition}

Rest mass $\mathrm{m}$ values obtained with (1) are marginal but certainly non-trivial, they vary from $7.373 \times 10^{-51}$ $\mathrm{kg} /$ atom for "relativistic" electron waveform, (not literature's $9.1094 \times 10^{-31} \mathrm{u}$ ) to americium's "relativistic" waveform $4.743 \times 10^{-41} \mathrm{~kg} /$ atom; these ultra-low values identify with 'absolute' atomic mass. Normally, theorists stop the investigation at this stage; i.e., experiment reveals that the atom has an unknown form with an ultra-low atomic mass value. Experiment also reveals that every atom defines with four 'rest' mass values. To our knowledge no attempt has been made to probe the origin of these rest mass variants called 'mass generations'. We do not intend to address the subject to any reasonable extent here, see, e.g., Obande (2019a; 2016b); briefly, the four generations comprise: the visible particulate form, denoted $m_{p}^{o}$; the waveform whose aggregate 
constitutes the CVF, $m_{w}^{*}$; invisible particulate matter equivalence of the waveform, $m_{p}^{*}$; and invisible particulate analogue of our visible form, $m_{p}^{\prime}$. All mass-energy actions(including, of course, metric space expansion MSE) are defined within the framework of the dynamics of spacetime matrices of these mass generations. The observational evidence that the CVF comprises exclusively aggregated atomic waveforms, Obande (2019a; 2016a) is particularly relevant here; given the elements' $v$ values, physics of the vacuum field reduces to classical mechanics. Newtonian mechanics analysis of the waveform reveals that: (i) the electron waveform and the photon are one and the same thing, Wall (2018), Obande (2016b); i.e., in line with decades of experimental results, the CVF is a photonic field, Williams and Mark (1997), Gauthier (2019), Atkins (2015); (ii) coincidence of the cosmic and atomic values of the ratio: matter energy density/vacuum energy density, $\sum m c^{2} / \sum h v=3.738 \times 10^{-23} / 3.6749 \times 10^{-23} \mathrm{Jatom}^{-1} \mathrm{CPU}^{-1}=1.0172$, indicates that visible reality is to particulate atom as the vacuum field is to the atomic waveform, Obande (2015a; 2019a); it also signifies remarkable internal consistency and affirms that the dynamics of the microcosm and macrocosm are quantifiable with same classical formalism; (iii) the fundamental physical constant FC is a coefficient of linear correlation of a specific vacuum field's physical parameter, Obande (2016a; 2017b); it re-affirms the physical essence of the vacuum field; (iv) remarkable similarity exists between electron configurations and morphologies of cosmological bodies indicative of existence of observational universal conformal invariance, Oldershaw (2014), Fedosin (2017), Wikiversity (2017); it supports our view that the classical and relativistic fields are analyzable with Newtonian mechanics thus, an investigation of atomic dynamics corresponds to one of the vacuum field dynamics and vice-versa, Obande (2017a); these positions inform the foundation of the present investigation.

\subsubsection{Geometric Structure}

The photon's geometric structure published by the team at Warsaw University is fundamental to the structure of quantum matter be it particulate or waveform, Chrapkiewicz et al. (2016), see also, Obande (2018). The structure consists of two counter rotating e-m cones or vortices/spirals joined at the apices, R\&R (1981, p. 90, Fig 12), Boldyreva (2018); it informs the structure of every object, Golubev (2016; 2014). We emphasize that the quantum vacuum is intrinsically periodic, R\&R (1981, p. 90, Fig.13); indeed, vacuum periodicity overrides all other large-scale field structure postulates, Khein (2006), Krasnoholovets (2016), Rafelski et al. (2009), Chernin (2001), Finkenstein (1996), Mau (1992), Dipert (1977). Quantum characteristics, ingrained in the atom as electronic configurations, dictate the course of every form of natural growth and ageing process including, of course, accelerating expansion of space.

\subsubsection{Physical Properties}

Contrary to age-old notion and accustomed impression, particulate matter has no intrinsic physical property; matter's physical property derives from the coefficient of linear correlation of its waveform harmonic oscillation $v_{w}$ parameters. This fact totally contradicts the consensus view, Schwinger (1973); however, the rather surprising position is not totally unfamiliar, Wheeler (1986), See (1920). The view as actively promoted by Lorentz holds that: "Indeed, one of the most important of our fundamental assumptions must be that the ether not only occupies all space between molecules, atoms or electrons, but that it pervades all these particles. We can reconcile with this, at first sight somewhat startling idea, by thinking of the particles of matter as of some local modifications [not 'excitations' but condensations] of the state of the ether ... I believe every physicist feels inclined to the view that all forces exerted by one particle on another, all molecular actions and gravity itself are transmitted in some way by the ether, so that the tension of a stretched rope and the elasticity of an iron bar must find their explanation in what goes on in the ether between the molecules," (our emphasis), Lorentz (1952, pp. 11,45,46). It means, of course, that particulate matter plays no role in MSE and we must seek the causality in physics of the vacuum field. The conventional vacuum is understood to include 'the quantum vacuum', 'Bose-Einstein condensate' and classical empty space in which transient energy packets forever pop in and out, Harnett (2014). Phenomena normally credited to field fluctuations such as: spontaneous photon emission; Lamb shift; Casimir force and cosmological perturbations, Milonni \& Smith (1975), Khosravi \& Loudon (1991), Reik et al. (2015), Mainland \& Mulligan (2020), all find superior explanations within the framework of CVF dynamics.

\section{Procedure}

The procedure has been published severally, from the element's $v$ and $m$ values we obtain other SHM parameters, mutual correlation of these yields a spectrum of coefficients many of which are torque fields, one of these motivates MSE, Obande (2019a; 2017b; 2016a). We conceive of three possible ways to investigate MSE within 
the framework of classical mechanics: (i) cosmic vacuum velocity-radius CVR gradient; (ii) simulated Hubble procedure SHP, and (iii) coupling to periodic distance scales, CPDS.

\subsection{Cosmic Vacuum Velocity-Radius CVR Gradient}

The torque field generated by the correlation,

$$
\rho_{w} / \sigma_{w}=8.51 \times 10^{-19}\left(\mathrm{rad} \mathrm{s}^{-1}\right)^{-2}
$$

describes an accelerated inverse angular momentum vector, an expansion, Obande (2015c; 2017b); dimensional analysis reveals space rotating with the tangential velocity,

$$
b \mathrm{v}_{t}=\mathrm{v}_{\mathrm{r}}=0.75 r \omega \mathrm{m} \mathrm{s}^{-1}
$$

In other words, vacuum energy density correlates with the stress field to motivate an invariant superluminal radial velocity $b v_{t}=v_{r}=0.75 \mathrm{r} \omega=7.064 \times 10^{8} \mathrm{~m} \mathrm{~s}^{-1}$, where $r, \omega, v_{t}, v_{r}$ are respectively, atomic radius, angular speed, tangential and radial velocities of the CVF and $\mathrm{b}=\tan ^{-1} \theta=0.75$ where $\theta=36.9^{\circ}$ is slope angle of the known universe. Established cosmic radius (distance length) $d_{\text {cosm. }}=14.26 \times 10^{3} \mathrm{Mpc}$ is, of course, the ultimate scale factor; given $v_{r}$ and $d_{c o s m}$, values an all-encompassing, representative, value of the vacuum velocity-distance coefficient retrieves with the simple expression,

$$
\begin{aligned}
H_{o \text { (calc.) }} & =v_{r} / d_{\text {cosm }} . \\
& =7.064 \times 10^{5} / 14.26 \times 10^{3}=49.5 \mathrm{~km} \mathrm{~s}^{-1} \mathrm{Mpc}^{-1}
\end{aligned}
$$

The value falls within the observational basket $H_{o}=100 \mathrm{hm} \mathrm{s}^{-1} \mathrm{Mpc}^{-1}$ where $h=0.5$ to 1

\subsection{Simulation of the Hubble Procedure}

Since (4) traces the Hubble constant to its primary (parametric) causality, the attempt to simulate the effect is not aimed at charting another $H_{o}$ evaluation route rather, the goal is to examine the velocity-distance profile for possible features that might highlight details of the phenomenon. We generate a 'variant' radial velocity field from the universal constant value according to the expression,

$$
v_{r}^{\prime}=v_{r(\text { reduced })}=v_{r(\text { cosm. })} \omega_{e(w)} / \omega_{A m(w)} k_{m ~ s}{ }^{-1}
$$

where $\omega_{\mathrm{e}(\mathrm{w})}$ and $\omega_{\mathrm{Am}(\mathrm{w})}$ are angular velocities of waveforms of atomic electron $\mathrm{e}^{-}$and americium Am, corresponding to lowest and highest $\omega$ values respectively; notably, the ratio $\omega_{e(w)} / \omega_{A m(w)}$ scales $v_{r}$ in accordance with the element's period and group. We adopt the same procedure to generate the distance scale $\mathrm{d}$ from $d_{\text {cosm. }}$, i.e.,

$$
d_{\text {cosm. }}^{\prime}=d_{\text {reduced }}=3.086 \times 10^{2} d_{\text {cosm. }} v_{e(w)} / v_{A m(w)} M p c
$$

where, $v_{e(w)}, v_{A m(w)}$ and $d_{c o s m}$. are e-m oscillation frequencies of $\mathrm{e}^{-}$and Am waveforms and the cosmic radius respectively. The choice of $\omega$ and $v$ to evaluate reduced $v_{r}$ and $d_{\text {cosm }}$ values is informed by the relations: $v=r \omega$ and $2 v_{A m(w)}=\left|12.89 \times 10^{9}\right| \equiv 13.7 \times 10^{9}$ yrs = age of universe, Obande (2017c; 2018). Using electron waveform values: $v_{e(w)}=1 \mathrm{~Hz} ; \quad v_{A m(w)}=6.44245 \times 10^{9} \mathrm{~Hz}$; $\omega_{e(w)}=6.283 \mathrm{rad} \mathrm{s}^{-1} ; \omega_{A m(w)}=4.048 \times 10^{10} \mathrm{rad} \mathrm{s}^{-1} ; d_{\text {cosm }}=4.4 \times 10^{23} \mathrm{~km} ; M p c=3.086 \times 10^{19} \mathrm{~km} ;$ $v_{r}=7.064 \times 10^{8} \mathrm{~ms}^{-1}$ we have,

$$
v_{r(e)}^{\prime}=7.064 \times 10^{5} \times 6.2832 /\left(4.048 \times 10^{10}\right)=1.096 \times 10^{-4} \mathrm{~km} \mathrm{~s}^{-1}
$$

and $d_{e}^{\prime}=4.4 \times 10^{23} /\left(6.44245 \times 10^{9} \times 3.086 \times 10^{19}\right)=2.22 \times 10^{-6} \mathrm{Mpc}$

hence, $H_{o}=1.096 \times 10^{-4} / 2.22 \times 10^{-6}=49.4 \mathrm{~km} \mathrm{~s}^{-1} \mathrm{Mpc}^{-1}$

It indicates that a valid theory of the vacuum field is absolutely indispensable to meaningful laboratory investigation of space expansion. Our so-called 'simulation' is simply another way of expressing the direct method, the two methods are, of course, expected to give same result. Values of $v_{E(w)}$ are presented in the Appendix; a sample of values of the variants $v_{r(E)}^{\prime}$ and $d_{E}^{\prime}$ are presented in Table 1 for illustration, and Fig 1 is the 'simulated' Hubble plot. 


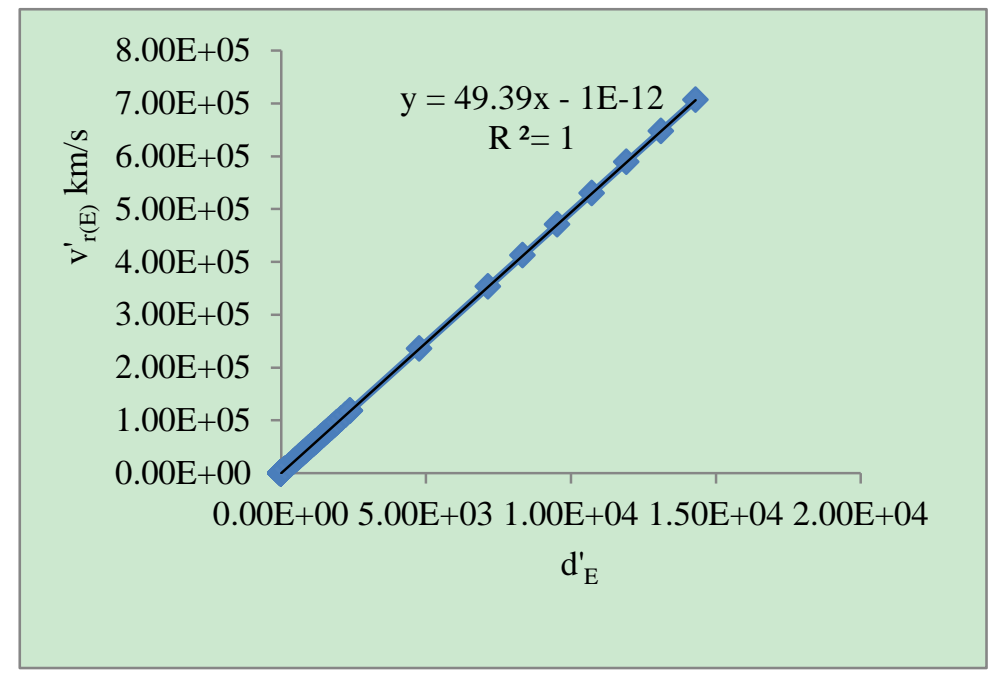

Figure 1. Vacuum field radial velocity-distance, $v_{r}^{\prime}$ and $d_{\text {cosm. }}^{\prime}$, plot

Table 1. Illustrated progression of $v_{\mathrm{r}}^{\prime} / \mathrm{km} \mathrm{s}^{-1}$ and $d_{\text {cosm. }}^{\prime} / \mathrm{Mpc}$ values across the chemical periodicity

\begin{tabular}{lllllllllllll}
\hline Atom & $e^{-*}$ & $\mathrm{Bt} *$ & $\mathrm{G} *$ & $\mathrm{H}$ & $\mathrm{He}$ & $\mathrm{Ne}$ & $\mathrm{Ar}$ & $\mathrm{Kr}$ & $\mathrm{Xe}$ & $\mathrm{Pb}$ & $\mathrm{Rn}$ & $\mathrm{Am}$ \\
\hline Mass No. & 1 & 12 & 20 & 24 & 28 & 36 & 44 & 62 & 80 & 108 & 112 & 121 \\
$\mathrm{~V}_{\mathrm{r}(\mathrm{E})}^{\prime} / \mathrm{km} \mathrm{s}^{-1}$ & $1.1 \mathrm{E}-04$ & $7.0 \mathrm{E}-03$ & $5.6 \mathrm{E}-02$ & $2.3 \mathrm{E}-01$ & $4.5 \mathrm{E}-01$ & $3.6 \mathrm{E}+00$ & $2.9 \mathrm{E}+01$ & $4.6 \mathrm{E}+02$ & $7.4 \mathrm{E}+03$ & $9.6 \mathrm{E}+04$ & $1.2 \mathrm{E}+05$ & $7.1 \mathrm{E}+05$ \\
$\mathrm{~d}_{\mathrm{E}}^{\prime} / \mathrm{Mpc}$ & $2.2 \mathrm{E}-06$ & $1.4 \mathrm{E}-04$ & $1.1 \mathrm{E}-03$ & $4.6 \mathrm{E}-03$ & $9.1 \mathrm{E}-03$ & $7.3 \mathrm{E}-02$ & $5.8 \mathrm{E}-01$ & $9.3 \mathrm{E}+00$ & $1.5 \mathrm{E}+02$ & $1.9 \mathrm{E}+03$ & $2.4 \mathrm{E}+03$ & $1.4 \mathrm{E}+04$ \\
\hline
\end{tabular}

*Unknown or invisible periods; three hidden periods precede H, Obande (2016b; 2013).

\subsection{Coupling to Periodic Distance Scales}

Space is periodic, evident from the wave-particle energy equation (1), $\phi h v=m c_{o}^{2}$. Notably, (1) equates not only energies but also geometric shapes (electronic configurations), of the wave and particulate forms of matter, Oldershaw $(2014 ; 2016)$, in other words, matter periodicity equates to spatial periodicity. Periodicity is unavoidable in quantum evolution, for one, it creates discontinuity, enabling a clear break between next neighbors without which the quantum state cannot be specified; on the other hand, it confers individuality and facilitates units and groups to be independently tailored and conferred with common 'quantum' characteristics such as charge and valence. The literature and our results indicate that these characteristics are actually intrinsic to the waveform (space) and their occurrence in the particle is emergent, Golubev (2016; 2014), Lorentz (1952). We see the recent observational evidence suggesting existence of spatial anisotropy, Migkas et al. (2020) as a timely evidence of what classical atomic physics, applied to cosmology/astrophysics, predicts. It reduces cosmology and astrophysics to atomic physics thus, successful quantification of atomic mass evolution AME should provide important clues to an atomic quantitative procedure for metric space expansion MSE. An earlier report of a successful procedure for AME reveals a logarithmic growth rate segmented at intervals by the noble gas, the layout corresponds to a universal periodicity comprising nine periods and nine groups, Obande (2015a; 2013). If the noble gas' inertness is structural in origin, i.e., the noble gas is a spacetime envelope totally devoid of tension, in spatial periodicity the waveform would be a structureless empty space regularly occurring between aligned cosmic structures. Reports of aligned cosmic structures, West (2017), Taylor \& Jagganathan (2016), Reiss et al. (2016), Crockett (2014) indicate existence of a spatial periodicity in which the galaxy takes the place of the chemical element in line with our earlier position, Obande (2017a, p.81).

The vacuum field coupling $r \omega=c_{o}$, manifests light, in other words, light is the tangential component of vacuum field angular speed. Interestingly, we see here that its interaction with the cosmic spacetime inclined at $36.9^{\circ}$ (to the horizontal) reveals the local transition from one logarithmic periodic spatial envelope to another, it manifests the observational effect associated with expansion of space, i.e.,

$$
H_{o}=r_{E(w)} \omega_{E(w)} / d_{E(w)}=v_{r(E)} / d_{E}
$$


where indices $\mathrm{E}$ and $\mathrm{w}$ refer to the chemical element and the waveform respectively. Notably, a most enlightening physics of the reality is embedded in the process summarized as light's "interaction with cosmic spacetime inclined at $36.9^{\circ}$ ". Until a more fundamental theory of the velocity-distance coupling is developed, (7) remains our best description of the phenomenon, the quantity $d_{E}$ accounts for the effect of periodicity to the finest of (elemental) details. Observe in Table 1 that $v_{r(E)}^{\prime}$ and $d_{E}^{\prime}$ increase individually in proportion to an increase in mass number in such a way as to guarantee $H_{o}$ invariance across the length of the cosmic radius; thus, observational multiplicity of the Hubble $a H_{o}$ must arise from effects other than temporal or random spatial variation.

\section{Results \& Discussion}

The results are presented in two figures and two tables, Figure 1 is a CVF v-d (Hubble) plot; Figure 2 is a schematic diagram of logarithmic spirals with varying numerical constant, $a H_{o}$; Table 1 highlights the correspondence between matter and spatial periodicities and Table 1A presents in the Appendix intrinsic atomic e-m frequencies of elements of the chemical periodicity, reproduced from Obande (2015a) to ease access.

A brief discussion of the Hubble constant was included in our preceding report, Obande (2019a), we regret to inform that, as is often the case with subjects we address in passing, most of that discussion was based on a hasty and sadly, wrong analysis. Notably, the equation describing space expansion

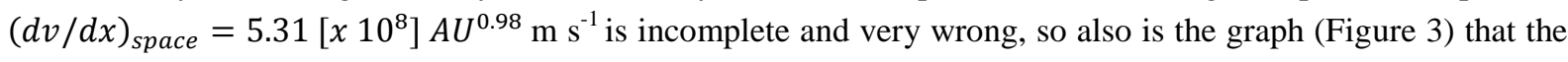
equation seeks to describe. The error is regretted.

\subsection{Chemical Principles in Atomic Physics}

A reviewer of our preceding article, Obande (2019b), took a particularly strong exception to the suggestion, implicit in our approach, that 'principles of chemistry' could conceivably be validly applied to atomic physics. The objection is premised on the presumption that these principles are emergent and "fully accounted for" in quantum electrodynamics, QED. To say the least, we find the view self-limiting. Since the 'principles' in question were not specified, we may assume that they likely include the foundations of our approach, foremost among which are: the unresolved question of deterministic nature of the chemical element's wavefunction; exclusive definition of the CVF with aggregate waveforms of the chemical elements; atomic mass concept with particular reference to the unspeakable question of a viable theory to account for absolute and relative atomic mass values; origin, and role of periodicity in nature with specific reference to the universal bipolar nature of reality responsible for division of matter into two diametric yet inseparable chiral periodic groupings, et cetera. Chemistry seems to get along pretty well with its traditional notions of these and other 'principles' but they remain thorny issues in particle physics. The objection claims that they have all been 'fully (?) accounted for' in QED, however, according to the literature, "In many ways, the 1964 quark hypothesis of particle physics is an ultimate concession to nature that the reductionist approach failed. For all practical purposes, quarks are quasi-particles (i.e., mathematical constructs), analogs of fractional charges known to condensed matter physicists working on fractional Hall effect phenomenon. ... The next unification attempts were made through the mathematics that came to be known as 'Special Relativity' by ...; which the author believes, has been a major misadventure and misdirection in physics since these efforts constituted efforts to eliminate the physical reality of space as ether in some form or other ..." Atkins (2015). We seek in our approach to demonstrate that modern physics' pressing tendency to relegate classical physics is informed primarily by a genuine difficulty with an analytical procedure for $v$ in (1). It restrains the equation's immense potential to the extent that absolute atomic mass remains supposedly indeterminate from first principles, the practice whereby particle physics relies on $m=E / c_{o}^{2}$ has been particularly limiting but, through our approach, absolutely avoidable see, Obande (2016b). 
Natural growth including, of course, spatial growth intersects the artificial boundaries of the core sciences, Golubev (2016; 2014), Yu CQ \& Rosenblatt (2007), Chin (2000). If our attempt could but draw theoretical physicists' attention to the deterministic (non-probabilistic) nature of the atomic wavefunction and liberate the rich physics embedded in the classical mass formula, our principal objective would have been more than realized.

\subsection{Expansion of Physical Space: I. The Hubble Constant}

Result of the present investigation is unambiguous; space is physical and its expansion is accelerating. However, an understanding of the "how?" would require a major shift away from long-held notions of reality. It would require a shift from the notion that the CVF consists of quantum fluctuations fleetingly popping in and out of existence eternally to the view which sees visible and invisible realities as different sides of the same coin; that, we believe, is what (1) has been telling us all along. The new view allows one to evaluate harmonic rotational parameters of physical space. Linear correlation of these parameters gives the fundamental physical constants, one of these, (2), reveals space radiating at the superluminal radial velocity $v_{r}=2.356 c_{o}$, it is responsible for metric space expansion MSE. Interestingly, with respect to radial motion of the photonic vacuum field, it is found that: "The superluminal energy quantum [SEQ] moves on the surface of a mathematical horn torus with a maximum speed $V_{\max }=c_{o} \sqrt{5}=2.236 c_{o}$ at the equator of the torus and a minimum speed $V_{\min }=c_{o}$ at the center point of the torus, Gauthier (2019). We find the coincidence between $v_{r}$ and $V_{\text {max }}$ values quite interesting, it reinforces our confidence in $v_{r}$, both values rely on SHM formalism but each takes a different route yet both arrive at same result. Of course, if all of space moved at same speed it wouldn't produce observational relative acceleration, as shown below, accelerated expansion of space results from $v_{r}$ coupling to logarithmic distance scales of quantized periodic space.

\subsubsection{Value of the Hubble Constant}

If the Hubble constant evaluated directly as quotient of two fundamental vacuum field constants, $H_{o(\text { calc. })}=$ $v_{r} / d_{\text {cosm. }}=49.5 \mathrm{~km} \mathrm{~s}^{-1} \mathrm{Mpc}^{-1}$, is not the true value, in our opinion, it must be the closest we have got. The value falls slightly short of qualifying for truly theoretical value on account only of the fact that our best theory of atomic mass, (1), is, as yet, semi-empirical. To have $H_{o(\text { calc. })}$ value fall within the observational basket speaks much in favor of credibility of our approach and, subject to independent verification, the value should qualify as standard for the observable universe. The graphical simulation, Fig. 1, is a device intended to explore details of the phenomenology, its replication of result of the direct method is naturally expected. Much more importantly, the device is able to reveal a bimodal cosmic space expansion profile typified by an initial compact gradient zone separated from a much more staggered terminal zone. Notably, identical bimodal feature is seen also in other parametric correlations, e.g., plots of stress $\tau$ vs. angular speed $\omega$, Obande (2017a; 2015b), it is indicative of radioactivity and electrical effects, Macken (2001). Briefly, the feature reveals a reality (atomic and cosmic) divided into two unequal e-m radiation spatial zones comprising an initial compact zone, covering 99.96\% of space and a terminal marginal slice covering the balance in which the familiar initial radiation transforms to radioactivity. We infer from the results that a cosmological analogue of radioactivity is in progress in space, it gives rise to the staggered data points at the latter half of Fig. 1. In this process, towards the terminal stages of the expansion dynamics, a cosmic quantum periodic envelope in the form of a stellar/galactic system or whole cluster, is ejected from the expanding cosmic spacetime envelope CSE. The atomic process is familiar but poorly understood but the cosmic process is as yet a curiosity. The two (atomic and cosmic) processes are governed by same quantitative formalism and we may hope that use of cosmological in place of atomic parameters as, e.g., Obande (2015b; 2017a), would lead eventually to an expansion of available options for quantitative astrophysics. With improvement in observation technology, a proof of this position would present as increased observation of ejection of massive (stellar) to supermassive (galactic) objects the more distant the CSE was from the observer. We would expect the frequency of observation of the phenomenon to be inversely related to observer's proximity to the boundary of the local universe, it is a closed universe see: 'The Static Sky', Obande (2017a); "Map of the entire night sky in X-ray", Williams (2019) and the "closed universe", Di Valentino et al. (2020). 
A slight digression: To our surprise, values of $v_{r}^{\prime}$ and $d_{\text {cosm. }}^{\prime}$. Table 1., initially thought artificial, provide incredibly useful insight into the question of spontaneity. An interesting theory of spacetime evolution can be built around the values but here we are able to present only a snapshot. In general, the values indicate a 'seeded' and slowly evolving universe but provide no clue regarding its origin. They ( $v_{r}^{\prime}$ and $d_{c o s m}^{\prime}$.) start at the origin of spacetime evolution from $1.1 \times 10^{-4} \mathrm{~km} \mathrm{~s}^{-1}$ and $2.2 \times 10^{-6} \mathrm{Mpc}$ respectively to constitute the 'seed'. After evolving through nine spatial periodic envelopes, demarcated by noble gases, R\&R (1981, p.90, Fig. 13) they achieve the respective terminal values $7.1 \times 10^{5} \mathrm{~km} \mathrm{~s}^{-1}$ and $1.4 \times 10^{4} \mathrm{Mpc}$ at the borders of visible reality. Notably, the 'seed' radius $2.2 \times 10^{-6} \mathrm{Mpc}=6.7892 \times 10^{16} \mathrm{~m}$, c.f. $\pi r_{e(w)}^{2}=7.067 \times 10^{16} \mathrm{~m}^{2}$, is in line with our position that visible reality is encased within electron waveform envelopes. It is, of course, quite possible but certainly premature to proceed to evaluate some other cosmological parameters through the use of $v_{E}^{\prime}$ and $d_{E}^{\prime}$; we find, for instance, that $14.26 \times 10^{3} / 2.2 \times 10^{-6}=6.48 \times 10^{9}$ initial spatial quantum units ISQUs approximates the present estimated size of our observable universe, $\left[v_{A m(w)} / v_{e(w)}=6.44 \times 10^{9} / 1.0=\right.$ $6.44 \times 10^{9}$, Obande (2018, p. 75)]; although 2ISQUs compare with present age of the universe, the new method would seem absolutely premature for wide digressions.

\subsection{Expansion of Physical Space: II. The Hubble Drift}

The logarithmic spiral is nature's structural member responsible for periodicity, see R\&R (1981, p. 90, Fig. 13). All will agree that the spiral is encountered in quite a wide variety of natural manifestations, Boldyreva (2018), Yu CQ \& Rosenblatt (2007), Chin (2000). We observed with curiosity in earlier studies that correlation envelopes of atomic harmonic parameters define exclusively with conic sections, Obande (2015c; 2019a); subsequent literature search revealed that the observation is an indication of intrinsically spiral (i.e. curved) spacetime posited originally by Descartes (1644) and formalized by Huygens (1690). The tangible chemical element condenses from its atomic waveform and achieves electrical neutrality as counter-rotating conjugate spiral pair inter-twined at the apex, R\&R (1981, p.90, Fig. 12); the shape informs spiral galaxies, the Whirlpool galaxy being the most conspicuous and in living matter, the shape is most noticeable among the gastropoda class of mollusks. It accounts for why most, probably all, natural growth processes are quantifiable with evolution of the logarithmic spiral. If, as we have shown, atomic mass evolves logarithmically, Obande (2017a, p.78; 2016b, p.93), see also Table 1A, spatial mass evolution should likewise be logarithmic; we submit, therefore, that what presents as MSE is the natural growth and ageing process of the cosmic vacuum field CVF, it leads eventually to exit from visible reality or death. The process is quantifiable with (8) and (9), Wikimedia.org,

$$
\begin{gathered}
r=a e^{k \theta} \\
d r / d \theta=a b e^{k \theta}=k r
\end{gathered}
$$

where $\mathrm{r}$ is the distance from the origin, $\theta$ is the $\mathrm{x}$-axis slope angle of the spiral taken from the origin, and a and $\mathrm{k}$ are constants. Comparison of (9) with (4) reveals that the cosmos is itself a spiral tilted at $\theta=36.9^{\circ}$ to the horizontal (a compelling phenomenological theory of light can be built on this revelation). Observe that, (8) and (9) are variants of Hubble's $d r / d t=H r$; thus, Descartes (1644) and Huygens (1690) were right all along physical space consists entirely of a spectrum of sizes of logarithmic spirals, the isolated forms give the vacuum the familiar description of a Dirac sea of electrons and positrons and the coupled form interprets as Dirac dipole sea.

It is clear from (8) and 9 that observational direct proportionality between vacuum radial velocity and distance length results from logarithmic nature of the CVF. In principle, the Hubble should have unlimited validity across the cosmic radius regardless of angular direction; however, over a given cosmic time interval $\mathrm{t}, H_{o}$ value would remain constant only if the velocity-time and distance-time gradients remained equivalent, i.e., $\Delta v_{r} / \Delta t \equiv$ $\Delta d / \Delta t$, a drifting Hubble, $a H_{o}$ or $H(t)$, is therefore an indication that $\Delta v_{r} / \Delta t \lessgtr \Delta d / \Delta t$. Since $v_{r}$ is invariant, $\left|v_{r}\right|=|r \omega|=\pi c_{o}$, Obande (2016a), the time-dependent distance scale $\mathrm{d} / d t$ remains the only possible causality of a drift. Fig. 2 is a diagram of logarithmic spirals with a common slope angle $\theta$ and varying constant a = 1, 2, 3, 4, 5, Wikimedia.org. Based on correspondence between (9) and (4), we posit that multiple values of the Hubble, $a H_{o}$, cannot be validly interpreted to hint at $H(t)$ but are indicative of cosmic multiplicity of universes. 


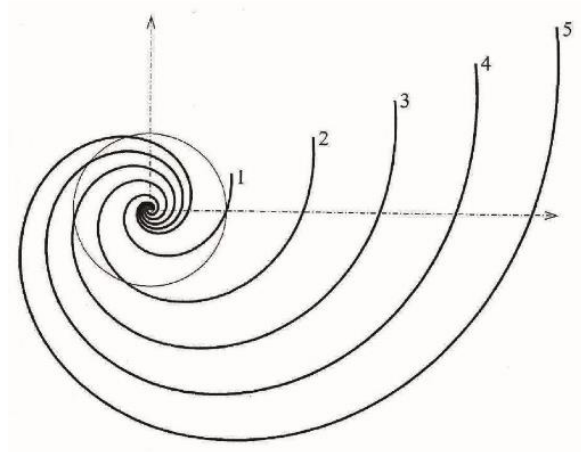

Figure 2. Spirals with slope angle $\alpha=20^{\circ}$ and $\mathrm{a}=1,2,3,4,5$.

Credit: Wikimedia.org.

The $L_{X}-T$ relation of galaxy clusters, Migkas et al. (2020), reveals a spatial "anisotropy" that manifests differential $H_{O}$ values occurring within the observational basket $H_{o}=100 \mathrm{~h} \mathrm{~km} \mathrm{~s}{ }^{-1} \mathrm{Mpc}^{-1}$ where the current values of $\mathrm{h}$ lie between 0.5 and 1.0. In our opinion, the report makes crucial fresh contributions to cosmology, it: (i) adds observational credence to the position that space is periodic and that multiple $H_{o}$ values do not necessarily reflect $H(t)$ or systematic error but likely point to existence of nested universes; (ii) reveals the existence of an erstwhile hidden contributor to causalities of $\mathrm{a} H_{o}$, where the numerical constant a, i.e., number of universes, might be as large as $10^{9}$ ! (iii) opens up a possibility for cosmological catalog of galactic clusters/universes using vacuum field periodicity VFP as boundary indicator; (iv) highlights the necessity for clarification of what constitutes the 'universe' as distinct from the 'cosmos' defined as summation of universes plus whatever else might be out there, (we believe that each universe is demarcated at its beginning by the red end and at its end by the blue end of the e-m spectrum); (v) might make the use of red-shift value for vacuum distance measure a lot more tricky than imagined unless a method existed to differentiate an intrinsic radiation due to the innate red end of a galaxy from that due to recession such as the authors" "a $\sim 5.5 \sigma$ anisotropy was obtained between the sky regions toward $(l, b) \sim\left(303^{\circ},-27^{\circ}\right)\left(H_{o} \sim 65 \mathrm{~km} / \mathrm{s} / \mathrm{Mpc}\right)$ and $\left((l, b) \sim\left(34^{\circ},+26^{\circ}\right)\left(H_{o}\right.\right.$ $\sim 77 \mathrm{~km} / \mathrm{s} / \mathrm{Mpc})$ ". Given our understanding of the results, we see the pairs of opposite diagonals as demarcating local universes.

According to the report, plots of best-fit $H_{o}$ values as a function of extragalactic sky position for $\theta=75^{\circ}$ cones for the ACC sample give the result $H_{o} / \mathrm{km} \mathrm{s}^{-1} \mathrm{Mpc}^{-1}=81,77,73,69,65,61$ and 57. Noting that $v_{r}$ is an invariant, the values correspond to cosmic radii values: $8.70 ; 9.15 ; 9.65 ; 10.20 ; 10.84 ; 11.55$, and 12.36 $\mathrm{Mpc}$ respectively. It indicates a structure in which an unknown number of younger clusters and/or universes is nested within our universe $d_{\text {cosm.. }}=14.26 \mathrm{Gpc}$. Thus, the NASA-NICER EXPT.'s X-ray map of the entire sky XMES, would be where to look for clues, Obande (2019a, p.27; Fig.2). XMES reveals the e-m skeleton of a small section of the sky, it consists of bright filaments, much like curved power lines interconnecting a number of seeming random distribution of much brighter power generating stations or spots. NASA explains that “... each bright spot represents an X-ray source while the bright filaments are their paths across the night sky". Observe from NASA's label of the galaxies that "the bright spots" are active galactic nuclei, the web of bright filaments inter-connect the AGNs revealing that reality is an integrated cosmic electromagnetic circuitry. Our present focus is on the nature of the unbroken filaments connecting the AGNs and the broken filaments that constitute the gritted background. It might take many more years of research to get to the bottom of what, in terms of clusters, galaxies or stars, constitute the bright filaments and the speckled background. Notably, observational $\mathrm{H}_{o}$ fits in well with XMES; the numerous e-m filaments and gritty background would tally with a steady-state universe if one assumed that reality was perpetually recycled; in which case there would be no permanent cosmological structures, reality would be an eternal spacetime matrix flux, matter would appear from 
the invisible, make its round and return to its source to reappear in perpetual re-cycles, NASA (2011). At any given point in space and time one would find a cocktail comprising evolving, matured, ageing and fading cosmological bodies exactly as we see around us and in the XMES. With advancement in technology, we may expect to find more, not less, drift in $H_{O}$ value as many more universes/clusters become accessible. The cosmos comprises nested universes - it is a plantation of rhizomes. In the course of literature review for final compilation of the results we chanced upon an on-going series of excellent studies of the subject by Masreliez (2001), we decided to publish our results nonetheless because we think the differences far outweigh the similarities in philosophy.

\subsection{A Steady-State Universe}

Observations thought to invalidate the steady-state theory were grossly misinterpreted. As shown here, metric space expansion does not arise from some explosion in or of spacetime, it arises from vacuum field density-stress $\rho_{w} / \sigma_{w}$ correlation. Likewise, the cosmic microwave background CMB is not a relic radiation from some past cosmic explosion, it is the zero-point radiation, i.e., radiation from the aggregated waveforms of atoms of the chemical elements, Obande (2019a). Observational lines of evidence in: the "Static Universe", Obande (2017a); the "X-ray map of the entire sky", NASA-NICER Experiment, Williams (2019) and "Planck Evidence for a closed universe ..." Di Valentino et al. (2020) individually and collectively discredit the "Big Bang' hypothesis, reveal a closed universe and indicate a steady-state universe. It seems, however, that the mainstream is unconvinced, it insists on a reality that complies with the Standard Model. The reaction is not entirely unexpected, it is understood that "The human mind treats a new idea the way the body treats a strange protein; it rejects it", Glorfeld (2020). As technology advances field work is likely to keep turning up evidence after evidence in support of the steady-state universe and, if no bias got in the way, it should eventually become unreasonable to deny the obvious.

The present result indicates that, as with many other areas of theoretical physics, issues with the steady-state theory trace to evolution of physics. Briefly, without the definitive $v$ value of the element's e-m oscillation, theoretical physics is in no position to handle physics of the vacuum field, the subject touches upon causality of (positive and negative) gravitation, vacuum energy density and the cosmological constant. All who have investigated the puzzle of constant energy density of the expanding cosmic vacuum field, Einstein (1917), MacMillan (1918), Jeans (1928), Bondi \& Gold (1948), Hoyle (1948) have reached the same obvious conclusion: "For the density to remain constant, new particles of matter must be continually formed within that volume from space", Einstein in O'Raifeartaigh et al. (2014). Jean's contribution to the subject is absolutely invaluable, he says, "The type of conjecture which presents itself, somewhat insistently, is that the centres of the nebulae are of the nature of 'singular points', at which matter is poured into our universe from some other spatial dimension ... so that they appear as points at which matter is poured into our universe from some entirely extraneous spatial dimension, so that to a denizen of our universe, they appear as points at which matter is continually created". This point of view, now about 100 years old, underscores researchers' common attitude to literature; there is no telling what amount of energy and resources recognition and observational probe of this viewpoint would have saved humanity. In the interval a new breed of physicists is insistently taking over with a seeming resolve to upturn the foundations. Nature designs the AGN to eternally circulate m-e matrices across the four phases of physical reality, $U_{p}^{o} \rightleftarrows U^{\prime}{ }_{p} \rightleftarrows U_{p}^{*} \rightleftarrows U_{w}^{*}$. It is a two-way valve, we cannot over-emphasize the fact, the AGN is not a singularity, indeed, nature posts no singularities, Obande (2018). Furthermore, there is no new creation of matter or space, the conservation law forbids it, "... mass is energy and neither can be created or destroyed" Roychoudhri (2015). Rather regrettably, the new physics has rid itself of the "non-conformal" energy conservation law. To confound the situation the list of promoters of this contrary doctrine number among the most colorful, the more recent advocates would include Parker (2012; 1969), Hawking (1975), Hashiba et al. (2020), Avdeenkov \& Zloshchatiev (2011). According to Kragh (2014), the new position assumes legitimacy from the view that, "After all, has energy conservation been proved experimentally to an indefinite accuracy". 
The question of expansion destination is straight forward, the universe is closed, Di Valentino et al. (2019), therefore it expands only locally. Expansion's destiny is, however, much more involved. Observe that mass flows in opposite directions along the two galactic spiral arms linked to the AGN; along one arm it flows into the AGN and along the other arm it flows out of the AGN into a seeming emptiness. The subject is expertly summarized for the layman in Britannica.com; within the limits of our search, we couldn't locate a Journal article on the subject. Absence of an article in the area wouldn't surprise because an AGN with two-way (in-and-out) mass flow would contradict the singularity doctrine. Materials flowing via the spiral arm into and out of the AGN likely experience different destinies. Every form of matter that comes into visible existence, including humans, eventually goes out of physical existence through self-expansion, expansion is an inescapable death ride. Matter that flows into the AGN gets transformed, transfigured and transited into an "entirely extraneous spatial dimension", and that which flows out into seeming emptiness, (i.e., the 'out-flows'), possibly constitutes the material mark-up that sustains constant energy density in an expanding volume, see Obande (2018). One expects that having an understanding of the purpose and phenomenology of the AGN should be as important, if not more so, than the current emphasis on its mechanics and thermodynamics, especially given the suggestion that it behaves more like electrodynamics than a thermodynamics system; it would seem, however, that modern physics places far less emphasis on phenomenology than the beauty that comes with mathematical speculation.

\subsection{How Is It All Going to End?}

Woollaston (2016) raises the 'mother' of all questions, how is it all going to end? Is the universe heading towards a Big Freeze, a Big Rip, a Big Crunch, or a Big Bounce? Or is it part of an infinitely recurring cyclic model? To this poser another source adds, "Will it tunnel into another universe altogether?" We do not, and cannot, claim to have the answer. We came across a most interesting account by Dyson (1979), although quite engaging, enlightening and immensely entertaining, it refers to an open universe. As shown here, our investigations consistently indicate a closed and remarkably stable eternal universe, a segment of a 4-in-1 grand cosmic design that voids continuous matter creation by an ingenuous device that 'pumps' m-e matrices through the cosmic structural constituents much like the heart pumps blood through the body's parts.

\section{Summary}

The study relies on the following earlier findings:

- The atom is a wave-particle conjugate comprising integral (co-existent) wave and particulate forms, the particle is the wave condensate, it is encased within the waveform;

- The atom is a harmonic e-m oscillator, its intrinsic physical properties result from the oscillation parameters;

- Matter field, or de Broglie radiation, $c^{o}=3.715352291 \times 10^{-14} \mathrm{~m} \mathrm{~s}^{-1}$ is inverse square of the vacuum field $c_{o}=2.99792458 \times 10^{8} \mathrm{~m} \mathrm{~s}^{-1}$ (actually, $\mathrm{rad} \mathrm{s}^{-1}$ ), i.e., $1063 \pi / c_{o}^{2}=c^{o}$.

- Material reality is encased within electron waveform envelopes, $r_{e(w)}=1.499 \times 10^{8} \mathrm{~m}$;

- The mass-energy equivalent formula $m c^{2}=h v$ is strictly applicable to only the vacuum field, the more general form is $\phi h v=m c_{o}^{2}$, it equates not only energies of the atom's wave and particulate forms, but also intrinsic geometries, i.e., electronic configurations, of both forms;

- Most, probably all, physical properties of matter arise from correlation of atomic waveform harmonic oscillation parameters, the correlation coefficient gives the fundamental physical constant while the correlation exponent gives the geometry (crystal planes) of the correlated envelope.

Based on the above positions, the results were analyzed to reveal that:

- The cosmic vacuum field CVF, i.e., space, shares identical quantization, periodicity and mass-evolution with matter; 
- The CVF is exclusively photonic, i.e., space is an aggregation of waveforms (e-m radiations) of the chemical elements; its geometric structure is as reported for the electron by Chrapkiewicz et al. (2016), it comprises two counter-rotating e-m conic or spiral force fields joined at the apices;

- Three possible methods were identified for classical investigation of the Hubble effect:

- direct division of intrinsic vacuum field velocity by the cosmic radius, $v_{r(\operatorname{cosm} .)} / d_{\text {cosm. }}$, CVR, this refers to the 'direct method';

- a simulation of the Hubble procedure SHP using values of the two cosmic invariants, $v_{r(\operatorname{cosm} .)}$ and $d_{\text {cosm. }}$. reduced in such a way as to retrieve the respective initial and final values; it, of course, gives an identical result with the direct method, i.e., $H_{o}=49.5 \mathrm{~km} \mathrm{~s}^{-1} \mathrm{Mpc}^{-1}$;

- Coupling to periodic distance scales CPDS, this is quantitatively identical to the SHP, conceptually, however, it relies on the fact that the chemical element's waveform is intrinsically periodic hence, at the level of the element $\mathrm{E}$, the coupling $v_{r} / d_{r(E)}$ translates as division of the invariant velocity field by a logarithmically decreasing distance scale; it gives rise to acceleration of a constant velocity field relative to space not time;

- Periodicity is indispensable to quantum evolution; it defines individuality of the evolving spacetime envelope thus allowing the envelope to be tailored to and conferred with specific quantum (geometric) characteristics such as valence and charge;

- If the noble gas' low reactivity was geometric in origin, its waveform would be a structureless empty space completely devoid of tension and regularly occurring between aligned cosmic diagonal structures in line with observation thus, in spatial periodicity the gas' waveform would demarcate cosmic structures;

- Light radiates as the tangential component of the vacuum field parametric coupling $r \omega=c_{o}$; its $36.9^{\circ}$ inclination $\left(\tan ^{-1} 0.75\right)$ couples to spatial periodicity to manifest accelerating expansion of space;

- Multiple values of the Hubble constant $a H_{o}$ is not likely due to experimental systematic error or drift over cosmic time scale, $\mathrm{H}(\mathrm{t})$, the effect is traceable to multiplicity of (smaller) universes nested within our universe;

- Mass ejection from a cosmic body is equivalent to (atomic) radioactivity, it signals ageing and approach of the body to disappearance from visible reality;

- Space is intrinsically spiral, i.e. intrinsically curved; it contains a series of quantum matter spiral envelopes whose radii varies from particulate americium's envelope $2.8 \times 10^{-24} \mathrm{~m}$ to $10^{5}$ light years for supermassive galaxies, it buttresses the Descartes-Huygens picture of the CVF. Spatial logarithmic spirality is responsible for periodicity and several features of light, including plane mirror image inversion;

- Result of the research reported by Migkas et al. (2020) is interpretable as indicative of observational spatial periodicity thus, it opens a possibility for compiling galactic catalog of the universe based on use of vacuum field periodicity VFP as demarcator;

- Reality is in an imperturbable (ideal) Steady-State, observations thought to invalidate this view were wrongly interpreted, accelerating expansion of space arises from multiple couplings of vacuum spacetime parameters, not a distant cosmic explosion, the cosmic microwave background is the zero-point vacuum radiation;

- The active galactic nucleus or black hole is not a singularity, it is a two-way valve that facilitates circulation of mass-energy matrices across the four phases or ref. frames of reality, there is no new creation of space or matter, only continuous recycling not unlike reproduction in the rhizome family.

\section{References}

Apell, D. (2008). The sun will eventually engulf earth - may be. Sci. Am. Retrieved from https://www.scientificamerican.com/article/the-sun-will-eventually-engulf-earth-m

Atkins, C. G. et al.. (2015). Are electrons oscillating photon, oscillating "vacuum", or something else? Panel discussion, Proc. SPIE Optical Eng. \& Appl. Nature of Light. Retrieved from https://eprints.gla.ac.uk/110969/1/110969.pdf

Avdeenkov, A. V., \& Zloshchatiev, K. G. (2011). Quantum Bose liquids with logarithmic nonlinearity: Self-sustainability and emergence of spatial extent. Retrieved from https://arxiv.org/pdf/1108.0847.pdf 
Boldyreva, L. B. (2018). Spin System of Physical Vacuum as a Source of Energy. Int. J. Phys. 6(4), 128. Retrieved from http://pubs.sciepub.com/ijp/6/4/5

Bondi, H., \& Gold, T. (1948). The steady-state theory of the expanding universe. Mon, Not. Roy. Ast. Soc. $108(3), 252$.

Brittannica.com. (2020). Earth in the Milky Way. Retrieved 3 October, from https://www.britannica.com/place/Milky-Way-Galaxy

Chernin, A. D. (2001). Reviews of Topical Problems: Cosmic Vacuum. Phys Uspekhi, 44(11), 1099. https://doi.org/101070/PU2001v044n11

Chin, G. J. (2000). "Organismal Biology: Flying Along a Logarithmic Spiral. Science, 290(5498), 1857. https://doi.org/10.1126/science.290.5498.1857c

Chrapkiewicz, et al.. (2016). Hologram of a Single Photon. Retrieved from arxiv.org/pdf/1509.02890.pdf

Crockett, C. (2014). Galaxies may be aligned across 1 billion light-years. Science News, 186(13), 6-6.

Descartes. (1644). Prinzipien Philosophie, Teil 3 von der Sichtharen Welt, Absmitt 23.

Di Valentino, E., Melchiorri, A., \& Silk, J. (2020). Planck evidence for a closed Universe and a possible crisis for Cosmology. Nature Astronomy, 4, 196. https://doi.org/10.1038/s41550-019-0906-9

Diaz, L. (2018). Africa is splitting into two continents - Here is why. Retrieved from https://the-conversation.com/africa-is-splitting-into-two-here-is-why/94056

Dil, E. (2016). Space creation mechanism during the expansion of the Universe. Adv. Astron. https://doi.org/10.1155/2016/4695065

Dipert, R. R. (1977). Peirce's Theory of the Geometric Structure of Physical Space. Isis, 68, 404.

Dyson, F. J. (1979). Time without end: Physics and biology in an open universe. Rev. Mod. Phys., 51(3), 447. https://doi.org/10.1007/BF02345020

Einstein, A. (1917). The Principle of Relativity (Dover, 1952), pp. 175-188. Translated from the original.

Fedosin, S. (2017). Infinite Hierarchical Nesting of Matter. Retrieved from https://en.wikipedia/wiki/Essays/Fedosin/Infinite_hierarchical_Nesting_of_Matter

Finkenstein, D. R. et al.. (1996). Hyper-crystalline vacua. Retrieved from https://arxiv.org/abs/quant-ph/9608024

Gauthier, R. (2019). Quantum-entangled superluminal double-helix photon produces a relativistic superluminal quantum-vortex zitterbewegung electron and positron, Part 2: Crossing light speed. J. Phys. Conf. Ser.1251012016. Retrieved from https://iopscienceiop.org/article/10.1088/1742-6596/1251/1/01201

Glorfeld, $\quad$ J. (2020). $\quad$ Quotes. $\quad$ Retrieved https://cosmosmagazine.com/biology/scienc-history/peter-medawar-solves-rejection

Golubev, S. N. (2014).The emergence and existence of life in the world of elements and the physical vacuum. Arch. Life Sci., 8(11), 1. https://doi.org/10.1016/jals.2014.11.1

Golubev, S. N. (2016). Structure mechanism of ordinary mass formation. J. Mod. Phys., 7, 875. https://doi.org/10.4326/jmp.2016.7909

Harnett, J. G. (2014). Expansion of space - A Dark Science. Ans. Res. J., 7, 453. Retrieved from http://www.answersingenesis.org/arj/v7/expansion- of-space.pdf

Hashiba, S., Yomada, Y., \& Yokoyama, J. (2020). Particle production induced by vacuum decay in real time dynamics. Retrieved from https://arxiv.org/pdf/2006.10986.pdf

Hawking, S. W. (1975). Particle creation by black holes. Comm. Math. Phys., 43(3), 199.

Hoyle, F. (1948). A new model for the expanding universe. Month. Not. Roy. Soc., 108, 372. Retrieved from http://adsabs-harvard.edu/abs/194MNRAS.108.372\#

Huygens, C. (1690). Cosmotheoros. Crom http://pasengenproject.com/blog16/2012/11/24/Christain-huydens-descartes-vortex

Jeans, J. (1928). Astronomy and Cosmogony. CUP. Cambridge.

Khein, R. M. (2006). A topological theory of the physical vacuum. Retrieved from https://www.22.pair.com/csdc/pdf/physicalvacuum.pdf 
Khosravi, H., \& Loudon, R. (1991). Vacuum field fluctuation and spontaneous emission in the vicinity of a dielectric. https://doi.g/10.1098/rspa.1991.0052

Kragh, H. (2014). Historical aspects of post-1850 cosmology. AIP Conf. Proc., 1632, 3. https://doi.org/10.1063/1.4902824

Krasnoholovets, V. (2016). Structure of Space and the Submicroscopic Deterministic Concepts of Physics. Apple Acad. Press. N. J. 08758.

Lorentz, H. A. (1952). The Theory of Electrons. Dover Publ. Inc. Haarlem, pp. 11, 34, 45.

Macken, A. J. (201). The universe is only space. Retrieved from http://onlyspacetime.com

MacMillan, W. D. (1918). On stellar evolution. ApJ., 48, 35.

Mainland, G. B., \& Mulligan, B. (2020). Polarization of Vacuum Fluctuations: Source of the Vacuum Permittivity and Speed of Light. Found. Phys., 50, 457. https://doi.org/10.1007/s10701-020-00339-3

Masreliez, C. J. (2001). The Scale Expanding Cosmos Theory and a New Conception of the Progression of Time. Astrophysical Ages and Time Scales. ASP Conf. Series, 245, 631. Retrieved from https://arxiv.org/pdf/1205.5616.pdf

Mau, R. V. (1992). Vacuum Structure and Matter Genesis. Workshop on QCD vacuum structure, Paris, June. Retrieved from http://inis.iaea.org/collection/NCLCollectionStore/_Public/25/004/19.pdf

Migkas, et al. (2020). Probing cosmic isotropy with a new X-ray galaxy cluster sample through the $L_{x}-T$ scaling relation. Retrieved from https://arxiv.org/abs/astro-ph/2004.03305

Milonni, P. W., \& Smith, W. A. (1975). Radiation reaction and vacuum fluctuation in spontaneous emission. Phys. Rev., A11, 814. https://doi.org/10.1103/PhysRevA.11.814

NASA.gov News. (2011). NASA's Hubble confirms that galaxies are the ultimate recyclers. Retrieved from https://nasa.gov/mision_pages/hubble/science/recyclers.html

Niederste-Osholdt, M. et al.. (2010). Alignment of brightest cluster galaxies with their host clusters. MNRAS, 405(3), 2023. https://doi.org/10.1111/j1365-2966.2010.16597.x

O' Raifeartaigh, et al.. (2014). Einstein's Steady-state theory: an abandoned model of the cosmos. Retrieved from https://arxiv.org/ftp/arxiv/papers/1402/1402.0132.pdf

Obande, O. P. (2013). Notes on Russellian cosmogony - Part 1: Absolute atomic mass. Int. J. Eng. Sci., 2, 68. Retrieved from http://www.theijes.com/papers/v2-i4/part.\%20(2)/M0242068077.pdf

Obande, O. P. (2015a). Notes on Russellian cosmogony. II. A procedure for evaluation of relative atomic mass and internal energy. Physics Essays, 28(1), 78.

Obande, O. P. (2015b). Classical mechanics analysis of the atomic wave and particulate forms. Int. J. Eng. Sci., 4(6), 1. Retrieved from http://www.theijes.com/papers/v4-i6/version-2/A046201011.pdf

Obande, O. P. (2015c). Classical Definitions of Gravitation, Electricity and Magnetism. Appl. Phys. Res., 7(6). https://doi.org/105539/apr.v7n6p85

Obande, O. P. (2016a). On the photon's identity: Implications for relativity and cosmology. Appl. Phys. Res., 8(5), 10. https://doi.org/105539/apr.v8n5p10

Obande, O. P. (2016b). Atomic mass: Origin, Units, and Constants. Appl. Phys. Res., 8(1), 92. https://doi.org/105539/apr.v8n1p92

Obande, O. P. (2017a). The Pan-STARRS1 Static Sky: A classical physics goldmine for cosmology. Appl. Phys. Res., 9(5), 75. https://doi.org/105539/apr.v9n6p75

Obande, O. P. (2017b). On the fundamental physical constants: I. Phenomenology. Appl. Phys. Res., 9(5), 42. https://doi.org/105539/apr.v9n5p42

Obande, O. P. (2017c). On the fundamental physical constants: II. Field coupling geometry. Appl. Phys. Res., 9(5), 62. https://doi.org/105539/apr.v9n5p62

Obande, O. P. (2018). A field concept of the black hole. Appl. Phys. Res., 10(4), 66. https://doi.org/105539/apr.v10n4p66

Obande, O. P. (2019a). Mass and energy densities of the cosmos. Appl. Phys. Res., 11(6), 19. https://doi.org/105539/apr.v11n6p19 
Obande, O. P. (2019b). On intrinsic Rotation of bodies. J. High Energy Phys. Grav. and Cosm., 5 , 868. https://doi.org/10.4236/jhepgc.2019.53043

Oldershaw, R. L. (2014). A surprising similarity between stars and atoms. Retrieved from https://www3.amherst.edu/ rlodershaw/stars2/index.html

Parker, L. (1969). Quantized fields and particles in expanding universes. Phys. Rev., 183, 1057.

Parker, L. (2012). Particle creation and particle number in an expanding universe.

Rafelski, J. et al.. (2009). Quantum Vacuum Structure and Cosmology. Retrieved from https://slac.stanford.edu/econf/C0906083/pdf/26.pdf

Reik, C., et al.. (2015). Direct sampling of electric - field vacuum fluctuations. Sci. Mag., 350(6259), 420. https://doi.org/10.1126/Science.aac9788

Reiss, A., et al.. (2016). A 2.4\% Determination of the local value of the Hubble constant. ApJ., $826,56$. https://doi.org/10.3847/0004-637X/826/1/56

Roychoudhri, C. (2015). The next level of unification: Waves and Particles as emergent oscillations of the same Complex Tension Field. Proc. SPIE 9570. The Nature of Light. Optics \& Applications, San Diego, 9-13 August. https://doi.org/10.1117112.2205311

Russell, W., \& Russell, L. (1981). Atomic Suicide? (2nd ed.). University of Science and Technology, Swannanoa, Waynesboro, Virginia, USA. Retrieved from https://www.scribd.com/doc/85626193/Atomic-Suicide-Walter-and-Lao-Russell

Schwinger, J. (1973). A Report on Quantum Electrodynamics. In The Physicist's Conception of Nature. (Dordrecht: Reidel), pp. 413-429.

See, T. F. F. (1920). New Theory of the Aether, $3^{\text {rd }}$ Paper. Astron. Nachr., 212(5079), 235. NASA Astrophysics Data System. Retrieved from https:// adsabs.harvard.edu/pdf/190AN...212 ..235S

Taylor, A. R., \& Jagganathan, P. (2016). Alignment of radio-galaxies in deep radio images of ELAIS N1. MNRAS Letters, 459(1), L36.

Wall, M. (2018). The Milky Way is growing faster than the speed of sound. Retrieved from https://www.space.com/40194-milky-way-galaxy-growing,html

West, M. J. et al.. (2017). Ten billion years of brightest cluster galaxy alignments. Nature Astronomy, 1(7), 0157.

Wheeler, J. A. (1986). How Come the Quantum?. Ann New York Acad. Sci. https://doi.org/10.1111/j.1749-6632.1986.tb12434.x

Wikimedia.org. $\quad$ Embedded Spirals. $\quad$ Retrieved $\quad$ October, $\quad 2020$, from https://wikipedia/commons/9/94/Spiral-log-a-1.5svg

Wikipedia.org, Logarithmic spiral. $\quad$ Retrieved $\quad$ October, $\quad 2020$, from https://en.wikipedia.org/wiki/Conical_spiral\#cite_note-5

Wikiversity. (2017). Similarity of matter levels. Retrieved from https://en.wikiversit/wiki/Similarity_of_matter_levels

Williams, J. G., \& Mark, M. B. (1997). Is the electron a photon with a toriodal topology?. Ann. Found. Louis de Broglie, 22(2), 133. Retrieved from http://cybso.org/electron.pdf

Williams, M. (2019). NASA is building up a map of the entire sky seen in X-rays, line by line with its NICER experiment.

Retrieved

from https://www.universe.com/142392/nasa-is-building-up-a-map-of-the-entire-sky-seen-in-X-rays-line-by-linewith-its-nicer-experiment

Wollaston, V. (2016). How will the universe end?. Retrieved from http://Wired.co.uk/article/how-will-univese-end

Yu CQ, C. Q., \& Rosenblatt, I. M. (2007). Transgenic corneal neurofluorescence in mice: a new model for in vivio investigation of nerve structure and regeneration. Invest Ophthalmol Vis, Sci., 48(4), 1535. 
Appendix 1A. Russell's Atomic e-m Frequencies v/Hz

\begin{tabular}{|c|c|c|c|c|c|c|c|c|c|c|c|c|c|c|c|c|c|}
\hline $\mathrm{A}^{*}{ }_{\mathrm{N}}$ & Atom & $\mathrm{N}$ & $A_{N}$ & Atom & $v$ & $\mathrm{~A}_{\mathrm{N}}$ & Atom & $v$ & $A_{N}$ & Atom & $\mathrm{N}$ & $\mathrm{A}_{\mathrm{N}}$ & Atom & $v$ & $\mathrm{~A}_{\mathrm{N}}$ & Atom & $v$ \\
\hline 1 & $\mathrm{e}$ & 1 & 21 & $\mathrm{Cg}$ & 512 & 41 & $\mathrm{P}$ & 163840 & 61 & $\mathrm{Br}$ & 3932160 & 81 & Cs & 67108864.0 & 101 & $\mathrm{Re}$ & 658887028.4 \\
\hline 2 & $\mathrm{Bl}$ & 2 & 22 & $\mathrm{D}$ & 1024 & 42 & $\mathrm{~S}$ & 196608 & 62 & $\mathrm{Kr}$ & 4194304 & 82 & $\mathrm{Ba}$ & 134217728.0 & 102 & Os & 689391057.5 \\
\hline 3 & Bs & 4 & 23 & $\mathrm{~T}$ & 1536 & 43 & $\mathrm{Cl}$ & 229376 & 63 & $\mathrm{Rb}$ & 4194304 & 83 & $\mathrm{La}$ & 201326592.0 & 103 & $\mathrm{Ir}$ & 719895086.5 \\
\hline 4 & A & 8 & 24 & $\mathrm{H}$ & 2048 & 44 & $\mathrm{Ar}$ & 262144 & 64 & $\mathrm{Sr}$ & 8388608 & 84 & $\mathrm{Ce}$ & 227137693.5 & 104 & $\mathrm{Pt}$ & 750399115.6 \\
\hline 5 & $\mathrm{Jm}$ & 8 & 25 & $\mathrm{~L}$ & 2560 & 45 & K & 262144 & 65 & $\mathrm{Y}$ & 12582912 & 85 & $\operatorname{Pr}$ & 252948795.1 & 105 & $\mathrm{Au}$ & 780903144.7 \\
\hline 6 & En & 16 & 26 & $\mathrm{Ha}$ & 3072 & 46 & $\mathrm{Ca}$ & 524288 & 66 & $\mathrm{Zr}$ & 16777216 & 86 & $\mathrm{Nd}$ & 278759896.6 & 106 & $\mathrm{Hg}$ & 811407173.8 \\
\hline 7 & Ey & 24 & 27 & $\mathrm{Ng}$ & 3584 & 47 & $\mathrm{Sc}$ & 786432 & 67 & $\mathrm{Nb}$ & 20971520 & 87 & $\mathrm{Pm}$ & 304570998.2 & 107 & $\mathrm{Tl}$ & 841911202.9 \\
\hline 8 & $\mathrm{Ah}$ & 32 & 28 & $\mathrm{He}$ & 4096 & 48 & $\mathrm{Ti}$ & 1048576 & 68 & Mo & 25165824 & 88 & $\mathrm{Sm}$ & 330382099.7 & 108 & $\mathrm{~Pb}$ & 872415232.0 \\
\hline 9 & $\mathrm{Bd}$ & 40 & 29 & $\mathrm{Li}$ & 4096 & 49 & $\mathrm{~V}$ & 1310720 & 69 & $\mathrm{Tc}$ & 29360128 & 89 & $\mathrm{Eu}$ & 356193201.2 & 109 & $\mathrm{Bi}$ & 872415232.0 \\
\hline 10 & $\mathrm{De}$ & 48 & 30 & $\mathrm{Be}$ & 8192 & 50 & $\mathrm{Cr}$ & 1572864 & 70 & $\mathrm{Ru}$ & 33554432 & 90 & $\mathrm{Gd}$ & 382004302.8 & 110 & Po & 939524096.0 \\
\hline 11 & $\mathrm{Rm}$ & 56 & 31 & $\mathrm{Be}$ & 12288 & 51 & $\mathrm{Mn}$ & 1835008 & 71 & $\mathrm{Rh}$ & 33554432 & 91 & $\mathrm{~Tb}$ & 407815404.3 & 111 & At & 1006632960.0 \\
\hline 12 & $\mathrm{Bt}$ & 64 & 32 & $\mathrm{C}$ & 16348 & 52 & $\mathrm{Fe}$ & 2097152 & 72 & $\mathrm{Pd}$ & 37748736 & 92 & Dy & 433626505.9 & 112 & $\mathrm{Rn}$ & 1073741824.0 \\
\hline 13 & $\mathrm{Mc}$ & 64 & 33 & $\mathrm{~N}$ & 20480 & 53 & Co & 2097152 & 73 & $\mathrm{Ag}$ & 41943040 & 93 & Ho & 459437607.4 & 113 & $\mathrm{Fr}$ & 1073741824.0 \\
\hline 14 & Pn & 128 & 34 & $\mathrm{O}$ & 24576 & 54 & $\mathrm{Ni}$ & 2359296 & 74 & $\mathrm{Cd}$ & 46137344 & 94 & $\mathrm{Er}$ & 485248708.9 & 114 & $\mathrm{Ra}$ & 2147483648.0 \\
\hline 15 & $\mathrm{Vt}$ & 192 & 35 & $\mathrm{~F}$ & 28672 & 55 & $\mathrm{Cu}$ & 2621440 & 75 & In & 50331648 & 95 & $\mathrm{Tm}$ & 511059810.5 & 115 & $\mathrm{Ac}$ & 3221225472.0 \\
\hline 16 & $\mathrm{Ou}$ & 256 & 36 & $\mathrm{Ne}$ & 32768 & 56 & $\mathrm{Zn}$ & 2883584 & 76 & Sn & 54525952 & 96 & $\mathrm{Yb}$ & 536872912.0 & 116 & $\mathrm{Th}$ & 3758096384.0 \\
\hline 17 & $\operatorname{Tr}$ & 320 & 37 & $\mathrm{Na}$ & 32768 & 57 & $\mathrm{Ga}$ & 3145728 & 77 & $\mathrm{Sb}$ & 54525952 & 97 & $\mathrm{Lu}$ & 536872912.0 & 117 & $\mathrm{~Pa}$ & 4294967296.0 \\
\hline 18 & $\mathrm{Bz}$ & 384 & 38 & $\mathrm{Mg}$ & 65536 & 58 & $\mathrm{Ge}$ & 3407872 & 78 & $\mathrm{Te}$ & 58720256 & 98 & $\mathrm{Hf}$ & 567374941.1 & 118 & $\mathrm{U}$ & 4831838208.0 \\
\hline 19 & $\mathrm{Hl}$ & 448 & 39 & $\mathrm{Al}$ & 98304 & 59 & As & 3407872 & 79 & I & 62014560 & 99 & $\mathrm{Ta}$ & 597878970.2 & 119 & $\mathrm{~Np}$ & 5368709120.0 \\
\hline \multirow[t]{2}{*}{20} & G & 512 & 40 & $\mathrm{Si}$ & $1 \mathrm{E}+05$ & 60 & $\mathrm{Se}$ & 3670016 & 80 & $\mathrm{Xe}$ & 67108864 & 100 & W & 628382999.3 & 120 & $\mathrm{Pu}$ & 5905580032.0 \\
\hline & & & & & & & & & & & & & & & 121 & $\mathrm{Am}$ & 6442450944.0 \\
\hline
\end{tabular}

$* \mathrm{~A}_{\mathrm{N}}=$ Nature's mass number (formerly, Russell's)

\section{Copyrights}

Copyright for this article is retained by the author(s), with first publication rights granted to the journal.

This is an open-access article distributed under the terms and conditions of the Creative Commons Attribution license (http://creativecommons.org/licenses/by/4.0/). 